\title{
ESTUDO COMPARATIVO DA GRANULOMETRIA DA LAMA VERMELHA DE BAUXITA VISANDO A APLICAÇÃO EM COMPÓSITOS DE MATRIZ POLIMÉRICA*
}

\author{
Paulo Victor Campos Sousa ${ }^{1}$ \\ Lesley Glenda Sales dos Santos ${ }^{2}$ \\ Verônica Scarpini Candido ${ }^{3}$ \\ Edinaldo José de Souza Cunha ${ }^{4}$
}

\section{Resumo}

Diante a atual preocupação com a disposição de resíduos industriais, diferentes alternativas vêm sendo desenvolvidas a fim de que hajam aplicações referentes a utilização desses resíduos. A lama vermelha, rejeito da lavagem da bauxita, cujo a disposição final estabelece-se dentro de grandes barragens, vem sendo alvo de inúmeras prospecções para uso. Deste modo, o presente trabalho visa estabelecer uma análise comparativa da granulometria da lama vermelha a partir da sedimentação hidráulica, valendo-se da Lei de Stokes e de ensaios em granulômetro visando conhecimento para a futura aplicação em polímeros. Sendo assim, foram desenvolvidos testes experimentais em proveta para gerar dados de processo para testes em maior escala. As amostras pós-sedimentação foram analisadas em um granulômetro Mastersizer 2000-HYDROG. Verificou-se que existe uma variação significativa no tamanho das partículas de lama vermelha, e que é possível proporcionar futuramente a formulação de compósitos a partir de cada faixa granulométrica.

Palavras-chave: Lama Vermelha; Rejeito da Lavagem da Bauxita; Polímero.

\section{COMPARATIVE STUDY OF THE GRANULOMETRY OF THE BAUXITE RED LAMA} LOOKING FOR THE APPLICATION IN POLYMERIC MATRIX COMPOSITES

\section{Abstract}

Given the current concern with the disposal of industrial waste, different alternatives have been developed in order to have applications regarding the use of this waste. Red mud, a waste from bauxite washing, whose final disposal is established within large dams, has been the subject of numerous surveys for use. Thus, the present work aims to establish a comparative analysis of the granulometry of the red mud from the hydraulic sedimentation, using the Stokes Law and granulometer tests aiming knowledge for the future application in polymers. Thus, experimental tests were developed in the test tube to generate process data for tests on a larger scale. The post-sedimentation samples were analyzed in a Mastersizer 2000-HYDROG granulometer. It has been found that there is a significant variation in the size of the red mud particles, and that it is possible to provide the composite formulation from each granulometric range in the future.

Keywords: Red Lama; Bauxite Washout Rejection; Polymers.

1 Graduando em Engenharia de Materiais, Faculdade de Engenharia de Materiais, Universidade Federal do Pará, Belém, Pará, Brasil.

2 Mestrado em engenharia química, PPGEQ - Programa de pós-graduação em Engenharia Química, Universidade Federal do Pará. Belém, Pará

3 Bióloga, Doutora em Ciência dos Materiais, Professora Adjunta I, Faculdade de Engenharia de Materiais, Universidade Federal do Pará (UFPA), Ananindeua-PA, Brasil

4 Doutor em Engenharia Química, Professor na Faculdade de Engenharia química e na Faculdade de Engenharia de Materiais, Universidade Federal do Pará, Belém, Pará, Brasil 


\section{INTRODUÇÃO}

A lama vermelha é um resíduo oriundo do refino da bauxita durante o processo Bayer para a produção da alumina e posterior produção do alumínio, sendo geralmente depositada em forma de lagoas (Manfroi, 2009). A indústria de extração do alumínio em 2008 foi responsável por gerar cerca de 120 milhões de toneladas por ano, em escala mundial, do resíduo conhecido como lama vermelha, material constituído por óxidos metálicos insolúveis dispersos em um meio altamente alcalino ( $\mathrm{pH} \geq 10$ ), (Resende, 2012).

Atualmente a tendência geral para resíduos industriais ou subprodutos, que são produzidos em países industrializados, é estudar formas alternativas para o seu aproveitamento, a fim de eliminar custos (Tsakiridis, 2004). Sendo assim, o rejeito da lavagem da bauxita tem sido alvo de amplos estudos, a fim de minimizar prejuízos ambientais e evitar danos às comunindades fronteiriças das indústrias de beneficiamento. $E$ também, o armazenamento desse material requer uma grande área para sua disposição, além de cuidados permanentes com a manutenção dessas áreas, o que acarreta um elevado custo às indústrias de refino da bauxita (Souza, 2008).

Segundo Pinheiro et al. (2015), a granulometria dos resíduos está relacionada com a mineralogia da rocha de origem, processo de extração, processamento e a forma como é despejada na barragem ou dique. Na faixa de curva granulométrica é impossível definir um padrão, pois as características como processamento, extração e mineralogia variam de região para região. De modo geral, a granulometria dos rejeitos de lama vermelha (LV) está próxima a dos síltes que varia de $10 \mu \mathrm{m}$ a $100 \mu \mathrm{m}$ (Villar, 2002). A análise granulométrica de partículas sólidas compreende a determinação do tamanho das mesmas, bem como da frequência com que ocorrem em uma determinada classe ou faixa de tamanho (Lima e Luz, 2001).

O trabalho tem como objetivo verificar e classificar as variações granulométricas da lama vermelha através da sedimentação gravitacional, e, construir uma análise comparativa a partir da Lei de Stokes e de ensaios por granulômetro a laser, visando a futura aplicação em compósitos de matriz polimérica.

\section{MATERIAIS E MÉTODOS}

\subsection{Ensaios de sedimentação}

A lama vermelha, inicialmente, foi submetida ao processo de desagregação. Nesse processo o material foi manualmente fragmentado utilizando-se uma peneira com abertura de 28 mesh Tyler $(0,600 \mathrm{~mm})$, para a desintegração do material. Os ensaios experimentais de sedimentação dão suporte para o cálculo de velocidade terminal de sedimentação, que por sua vez, permite a separação em três faixas de granulometria perceptíveis no procedimento.

Sedimentação em proveta: Para a realização do ensaio em proveta foi utilizado um volume de $1 \mathrm{~L}$ de água destilada para $100 \mathrm{~g}$ de amostra e o auxílio de uma escala milimetrada e de um cronógrafo para a determinação do tempo de sedimentação e a altura de cada camada formada. Ao adicionar-se o resíduo, imediatamente faz-se a 
agitação da mistura e ao colocar a proveta sobre a bancada o cronógrafo é acionado para a contagem do tempo de sedimentação das camadas formadas anotando-se as respectivas alturas. Deste modo, foram realizados quatro testes em proveta para se obterem dados regulados de procedimento, fazendo um levantamento estatístico, que definiram parâmetros do processo.

Sendo assim, tornou-se possível obter dados para calcular a velocidade terminal de sedimentação das partículas através da Lei de Stokes para cada camada e consequentemente calcular também os diâmetros referentes a estas partículas, a fim de se determinar suas granulometrias e, também, estimar os valores para aumento de escala.

Sedimentação em maior escala: Para a realização do processo em maior escala foi utilizado um volume de $6 \mathrm{~L}$ de água destilada para $600 \mathrm{~g}$ de amostra. Com base nas velocidades de sedimentação obtidas no ensaio em proveta foi possível inferir o tempo para separação do material sedimentado, através da transferência da polpa sobrenadante para outro recipiente com auxílio de um sifão. O material sedimentado restante no recipiente após a transferência foi seco em estufa por 24 horas com temperatura constante de $100^{\circ} \mathrm{C}$.

\subsection{GRANULOMETRIA A LASER}

A granulometria a laser foi realizada com o auxílio de um granulômetro Mastersizer 2000-HYDROG, onde foram feitas análises para amostras secas do rejeito antes da sedimentação e também para as três camadas formadas no processo de sedimentação gravitacional.

\section{RESULTADOS E DISCUSSÃO}

\subsection{Ensaios de Sedimentação}

Após os ensaios de sedimentação em proveta obtiveram-se os dados referentes ao tempo de formação, a altura do líquido e altura da primeira e segunda camada que estão expressos na Tabela 1. A terceira camada não apresentou dados de altura e tempo de sedimentação devido ela ser composta por partículas extremamente pequenas que ficavam suspensas no líquido, portanto, convencionou-se que a terceira camada é definida por estes particulados em suspensão. 
Tabela 1 - Dados da sedimentação hidráulica

\begin{tabular}{c|c|c}
\hline Camada & $\begin{array}{c}\text { Tempo } \\
(\mathbf{s})\end{array}$ & $\begin{array}{c}\text { Altura } \\
(\mathbf{m m})\end{array}$ \\
\hline \multirow{5}{*}{ Primeira } & 75 & 11,1 \\
& 62 & 9 \\
& 54 & 9 \\
& 55 & 13 \\
\hline \multirow{5}{*}{ Segunda } & 541,8 & 10,45 \\
& 1050 & 6 \\
& 1080 & 10 \\
& 720 & 7 \\
\hline
\end{tabular}

Conforme os dados verificados na Tabela 2, referentes ao tempo de formação e a altura de cada camada, calculou-se a velocidade terminal (VT) das partículas das duas camadas obtidas primordialmente. A partir de valores obtidos do parâmetro $\mathrm{Cd} / \mathrm{Re}$ definido através da Equação 1, e considerando a esfericidade das partículas (Ф) igual a 1, determinou-se o número de Reynolds e o regime de escoamento para cada camada, o que torna possível estabelecer as equações para o cálculo do diâmetro médio das partículas tendo-se como princípio o ábaco $\mathrm{Cd} / \operatorname{Re} \times \operatorname{Re} \times \Phi$.

$$
\frac{C d}{\operatorname{Re}}=\frac{4 \cdot\left(\rho_{s}-\rho_{f}\right) \cdot g \cdot \mu f}{3 \rho_{f}^{2} \cdot v^{3}}
$$

Tabela 2 - média dos dados de tempo e altura nos testes de sedimentação

\begin{tabular}{c|c|c}
\hline Camada & $\begin{array}{c}\text { Tempo Médio } \\
(\mathbf{s})\end{array}$ & $\begin{array}{c}\text { Altura média } \\
\text { percorrida pelas } \\
\text { partículas (mm) }\end{array}$ \\
\hline LVB & 0 & 195,75 \\
Primeira & 61,5 & 185,225 \\
Segunda & 847,95 & 187,388 \\
\hline
\end{tabular}

Sendo assim, pôde-se verificar que a primeira camada, de acordo com seu número de Reynolds, está dentro do regime transitório, já a segunda cama está definida dentro do regime laminar. Com estas definições tornou-se possível calcular o diâmetro médio das partículas (Dp) da primeira camada de acordo com a Equação 2 e da segunda camada de acordo com a Equação 3, ambas relacionando a velocidade terminal (VT) com o diâmetro das partículas.

$$
D_{p}=\sqrt[1,14]{\frac{V_{T} \rho_{f}^{0,129} \mu_{f}^{0,43}}{0,153 G^{0,71}\left(\rho_{p}-\rho_{f}\right)^{0,71}}}
$$




$$
D_{p}=\sqrt[2]{\frac{V_{T} 18 \mu_{f}}{G\left(\rho_{p}-\rho_{f}\right)}}
$$

Onde: $D_{p}$ é o diâmetro da partícula; $V_{T}$ é a velocidade Terminal $(\mathrm{m} / \mathrm{s})$, $G$ é a aceleração da gravidade $(9,81 \mathrm{~N} / \mathrm{Kg}), \rho_{\mathrm{f}}=$ massa específica da água $\left(10^{3} \mathrm{Kg} / \mathrm{m}^{3}\right), \rho_{p}$

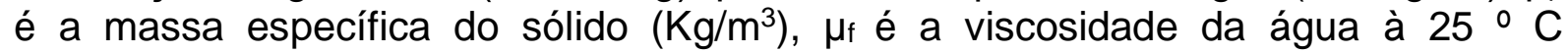
$\left(0,890 \times 10^{-3} \mathrm{~N} . \mathrm{s} / \mathrm{m}^{2}\right)$.

Os resultados obtidos para a velocidade terminal foram $3,012 \times 10^{-3} \mathrm{~m} / \mathrm{s}$ e $2,21 \times 10^{-4}$ $\mathrm{m} / \mathrm{s}$ para a primeira e segunda camada respectivamente. Já o diâmetro médio das partículas obtido para a primeira camada foi de $1,37 \times 10^{-5} \mathrm{~m}$ e para a segunda camada foi de $1,7 \times 10^{-5} \mathrm{~m}$. Estes valores demonstram que a primeira camada possui partículas relativamente maiores que as partículas da segunda camada, justificando o seu tempo de sedimentação, levando em consideração também o fato de que a terceira camada requer um tempo mais longo para a sedimentação, tendo suas partículas em suspensão, o que não acontece com a segunda camada, que pode ser observada minutos depois da formação da primeira camada.

A sedimentação em maior escala possibilitou a produção das três camadas em maiores quantidades, seguindo o mesmo procedimento da sedimentação em proveta, para que sejam inseridas na produção de placas de compósitos.

\subsection{Granulometria a Laser}

Os resultados de granulometria a laser para as camadas, incluindo a terceira camada, que após secagem pôde ser analisada no granulômetro, estão expressos na Figura 1.

Figura 1 - Comparativo dos resultados de granulometria a laser

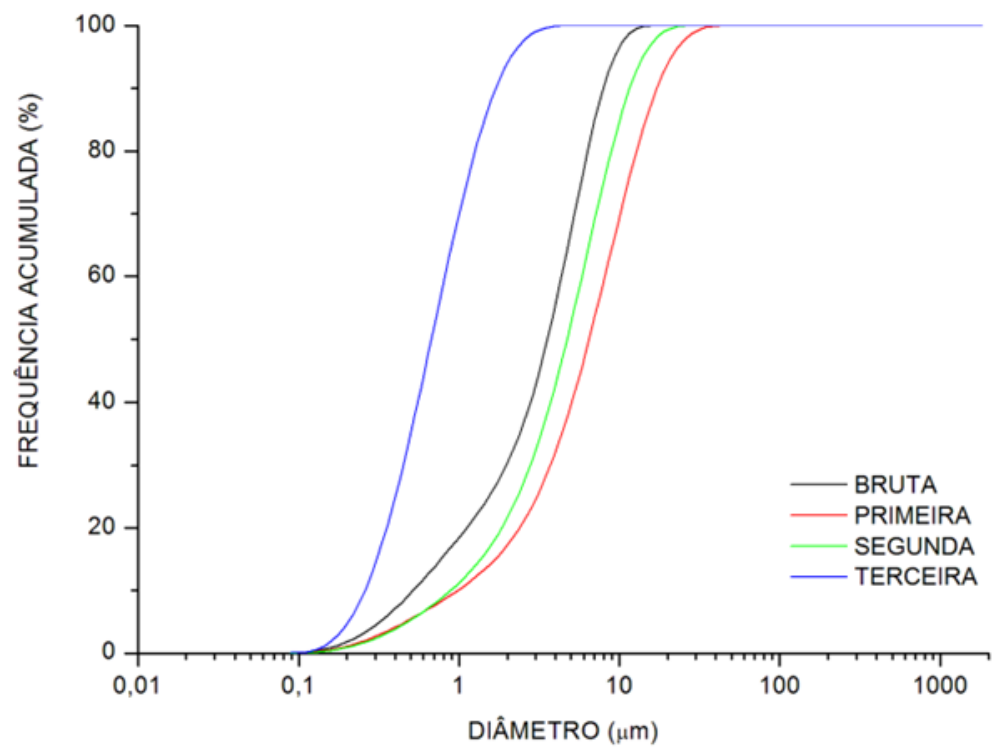

Os testes de granulometria a laser foram pertinentes aos resultados obtidos pela Lei de Stokes para o diâmetro médio das partículas de cada camada de lama vermelha após sedimentação. A partir da Figura 1, onde são expressos de maneira comparativa, os resultados da granulometria a laser do resíduo antes e após sedimentação, pôde-se notar que a terceira camada, que apresentou $D_{50}$ igual a $0,67 \mu \mathrm{m}$, possui partículas com diâmetros inferiores às da primeira e segunda 
camada pós-sedimentação que obtiveram diâmetros médios de 6,47 $\mu \mathrm{m}$ e 4,71 $\mu \mathrm{m}$ respectivamente.

Percebe-se também, que a granulometria da lama vermelha bruta está em um perímetro médio entre as curvas de granulometria das três camadas. Nota-se assim, que a primeira camada sedimentada possui partículas razoavelmente maiores do que a segunda camada, que por sua vez, tem partículas maiores do que as da terceira camada de lama vermelha. Segundo Lima e Luz (2001) as partículas podem ter várias formas, que influenciam determinadas propriedades, tais como fluidez, empacotamento, interação com fluidos e poder de cobertura de pigmentos.

\section{CONCLUSÃO}

Os ensaios granulométricos, a partir da sedimentação gravitacional da lama vermelha, caracterizaram o tamanho das partículas de acordo com três faixas granulométricas, indicando que, a partir da lei de Stokes, partículas da terceira camada possuem menores diâmetros. Denota-se assim, que a separação granulométrica a partir da sedimentação gravitacional é uma técnica eficiente, visto que a velocidade terminal de sedimentação está diretamente ligada ao diâmetro das partículas.

Nota-se que a lama vermelha em estado bruto apresenta uma média das três camadas observadas na sedimentação, e também que existe uma variação significativa no tamanho das partículas do resíduo. Deste modo, uma futura aplicação em polímeros poderá gerar compósitos com propriedades distintas quando relacionarem-se os diferentes tamanhos das partículas de lama vermelha.

\section{REFERÊNCIAS}

1 LIMA, R. M. F.; LUZ, J. A. M. Análises granulométricas por técnicas que se baseiam na sedimentação gravitacional: Lei de Stokes. Rev. Esc. Minas. vol.54 no.2 Ouro Preto, 2001.

2 MANFROI, I. C. Avaliação da lama vermelha como material pozolânico em substituição ao cimento para produção de argamassas. 2009. Dissertação (mestrado), Programa de Pós-Graduação em Engenharia Civil - Universidade Federal de Santa Catarina.

3 PINHEIRO, E. R.; FERNANDEZ, O. J. C.; COSTA, J. C. Química-Mineral das camadas inertizadas em lama vermelha da Hydro ALUNORTE. 22을 CBECiMat. 2016.

4 PINHEIRO, E. R.; FERNANDEZ, O. J. C.; COSTA, J. C. Química-Mineral das camadas inertizadas em lama vermelha da Hydro ALUNORTE. 22ํㅡㄹ CBECiMat. 2016.

5 SOUZA, W. B. de. Estudo da adição de lama de bauxita em polímero de poli(metacrilato de metila) por meio de polimerização em massa e moldagem em casting. 2008. Dissertação (Mestrado em Ciência de Tecnologia nuclear-Materiais) Instituto de Pesquisas Energéticas Nucleares, São Paulo, 2008.

6 TSAKIRIDIS. P. E.; AGATZINI-LEONARDOU. S.; OUSTADAKIS. P. Red mud addition in the ra1 meal for the production of portland cement clinker. Journal of Hazardous Materials. v. 116. p. 103-110, 2004

7 VILLAR, L. Estudo do adensamento e ressecamento de resíduos de ineração e processamento de bauxita. 2002, Tese (Doutorado) - Programa de Pós-Graduação em Engenharia Civil do Departamento de Engenharia Civil do Centro Técnico Científico da PUC-Rio. 